\title{
The diversity of soft X-ray spectra in quasars
}

\section{Citation}

Elvis, M., B. J. Wilkes, and H. Tananbaum. 1985. "The Diversity of Soft X-Ray Spectra in Quasars." The Astrophysical Journal 292 (May): 357. doi:10.1086/163166.

\section{Published Version}

$10.1086 / 163166$

\section{Permanent link}

http://nrs.harvard.edu/urn-3:HUL.InstRepos:32955251

\section{Terms of Use}

This article was downloaded from Harvard University's DASH repository, and is made available under the terms and conditions applicable to Other Posted Material, as set forth at http:// nrs.harvard.edu/urn-3:HUL.InstRepos:dash.current.terms-of-use\#LAA

\section{Share Your Story}

The Harvard community has made this article openly available.

Please share how this access benefits you. Submit a story.

Accessibility 
The Astrophysical Journal, 292:357-361, 1985 May 15

(C) 1985. The American Astronomical Society. All rights reserved. Printed in U.S.A

\title{
THE DIVERSITY OF SOFT X-RAY SPECTRA IN QUASARS
}

\author{
Martin Elvis, Belinda J. Wilkes, and Harvey TANanbaum \\ Harvard-Smithsonian Center for Astrophysics \\ Received 1984 September 14; accepted 1984 November 29
}

\begin{abstract}
We present Einstein imaging proportional counter spectra for three quasars (NAB 0205+024, B2 $1028+313$, PG $1211+143$ ). These spectra cover the soft X-ray band $0.1-4.0 \mathrm{keV}$. Power law fits to these spectra have best-fit energy indices of $1.2_{-0.2}^{+0.6}, 0.6_{-0.2}^{+0.3}$, and $2.2 \pm 0.4(90 \%$ confidence errors) respectively. They show no evidence for low-energy absorption in excess of the galactic values. Limits on any excess column of cold matter are $\lesssim 3 \times 10^{20}$ atoms $\mathrm{cm}^{-2}$.

These diverse power-law slopes contrast strongly with the suggestion of a uniform 0.7 value for Seyfert galaxies at higher energies $(2-100 \mathrm{keV})$. The implications of this diversity for (i) the X-ray background, (ii) X-ray continuum emission mechanisms, and (iii) the production of the optical/UV emission lines are discussed briefly.

Subject headings: quasars - radiation mechanisms - X-rays: sources
\end{abstract}

\section{INTRODUCTION}

Quasars are widely recognized as powerful X-ray sources. Almost 400 quasars have been detected as X-ray sources (Tananbaum et al. 1979; Zamorani et al. 1981; Ku, Helfand, and Lucy 1980; Margon, Chanan, and Downes 1982; Reichert et al. 1982; Kriss and Canizares 1982; Gioia et al. 1984; Stocke et al. 1983; Tananbaum et al. 1983) by the Einstein Observatory imaging proportional counter (IPC, Giacconi et al. 1979; Gorenstein, Harnden, and Fabricant 1981). Their X-ray emission is an important parameter in determining the total luminosity (Lightman 1981), continuum emission mechanisms (Rees 1981), and production of the prominent optical and UV emission lines that are characteristic of these objects (Kwan and Krolik 1981). It also seems likely that quasars are major, if not dominant, contributors to the diffuse X-ray background (Maccacaro, Gioia, and Stocke 1984; Marshall et al. 1984). To understand more reliably the role played by the X-ray emission requires at least some knowledge of the spectra in the X-ray band. Unfortunately, very few X-ray spectra exist for quasars, since all but a handful are too faint to be detected with nonimaging instruments.

For Seyfert galaxies, there are about 30 with published 2-20 keV spectral data (Mushotzky 1984). These spectra show a striking uniformity. Apart from a tendency for lower luminosity objects to show large low-energy absorption (Lawrence and Elvis 1982; Mushotzky 1982), they are all well fitted by a single power law of energy index $\alpha_{E}=0.7\left(F_{v} \propto v^{-\alpha}\right)$. Where measured, the data are consistent with the same power law extending up to $120 \mathrm{keV}$ (Rothschild et al. 1983). A similar result has been found at lower energies, $0.75-4.5 \mathrm{keV}$, with the Einstein solid state spectrometer (Petre et al. 1984). Also, for a sample of 25 quasars, Worrall and Marshall (1984) derive a co-added $\sim 1$ to $\sim 10 \mathrm{keV}$ power-law energy slope of $0.8_{-0.3}^{+0.2}$, if $N_{\mathrm{H}}$ is held below $3 \times 10^{20}$ atoms $\mathrm{cm}^{-2}$.

Below some energy near $1 \mathrm{keV}$, active galaxy continua must turn upward in order to meet the ultraviolet continuum, which typically has $\sim 100-1000$ times the flux density of the X-ray continuum. Pravdo et al. (1981) have reported a steep $\left(\alpha_{E}>2\right)$ low-energy X-ray spectrum in the low-energy X-ray selected Seyfert galaxy $\mathrm{H} 1613+06$. This may provide an example of such a turn-up. However, Petre et al. (1984) find that the typical upturn energy for Seyfert 1 galaxies must lie below 0.7 $\mathrm{keV}$.

Most discussions of quasar X-ray emission have thus assumed a hard $\alpha=0.7$ spectrum. Several theoretical attempts have been made to explain this "universal " spectrum (Mezaros 1983; Schlossman, Shaham, and Shaviv 1984; Kazanas 1984). Our new Einstein IPC spectra do not support such a simple picture.

The recent calibration of the IPC (Harnden and Fabricant 1985) now makes it possible to determine the instrument gain accurately for point sources on axis. This removes the major uncertainty involved in using the intrinsic energy resolution of the IPC to generate spectral fits. The IPC data base is currently being reprocessed ("Rev. 1" processing, Harnden et al. 1984) to make use of this new calibration, and this is leading to an increasing pool of X-ray spectral data.

Since a large number of quasars were observed on axis with the IPC, we have started a program to determine their soft $\mathrm{X}$-ray spectra. The first results from this program clearly demonstrate a wide variety of spectral slopes in the $0.1-4.0 \mathrm{keV}$ band. In this paper results for three of the first quasars to be processed are reported. They were chosen to illustrate the diversity we are finding in the spectral slope.

\section{DATA ANALYSIS}

The energy resolution of the IPC is only $\sim 100 \%$ at $1 \mathrm{keV}$ (Gorenstein, Harnden, and Fabricant 1981). However, its bandpass of more than one decade is enough to allow spectral determinations to good accuracy (e.g., $\pm 0.2-0.3$ in power-law energy index, Harnden et al. 1984). This bandpass (0.1-4.0 keV) is interrupted by a carbon absorption edge at $0.3 \mathrm{keV}$ due to the material in the counter window (Gorenstein, Harnden, and Fabricant 1981). We have found that the transmitted flux below this edge is essential to obtaining fits with reasonable uncertainties. Thus sources with a total hydrogen equivalent absorbing column of $10^{21}$ atoms $\mathrm{cm}^{-2}$ or more will not be well constrained. For this reason we have selected on-axis objects

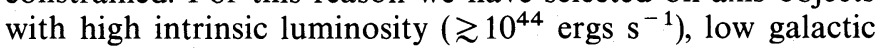


TABLE 1

EINSTEIN IPC OBSERVATIONS

(Rev. 1 Processing)

\begin{tabular}{|c|c|c|c|c|c|c|c|c|}
\hline Quasar & $z$ & $m_{B}$ & $\begin{array}{l}N_{\mathrm{H}}(\text { galactic) } \\
\text { (atoms cm }^{-2} \text { ) }\end{array}$ & $\begin{array}{l}\text { Sequence } \\
\text { No. }\end{array}$ & $\begin{array}{l}\text { Observation } \\
\text { Date }\end{array}$ & $\begin{array}{l}\text { Detector }^{\mathrm{b}} \\
\text { Gain }\end{array}$ & $\begin{array}{c}\text { Exposure } \\
\text { Time } \\
\text { (s) }\end{array}$ & Net Counts ${ }^{c}$ \\
\hline NAB $0205+024$ (Mrk 586). & $0.155^{\mathrm{d}}$ & $15.4^{\mathrm{d}}$ & $2.4 \times 10^{20}$ & 3978 & 1979 Jul 20 & 13.7 & 7608 & $1410 \pm 41$ \\
\hline B2 $1028+313 \ldots \ldots \ldots \ldots \ldots \ldots$ & $0.177^{\mathrm{e}}$ & $17.07^{\mathrm{e}}$ & $1.0 \times 10^{20}$ & 4256 & 1979 May $24 / 25$ & 11.8 & 6595 & $1303 \pm 40$ \\
\hline PG $1211+143 \ldots \ldots \ldots \ldots \ldots \ldots \ldots$ & $0.085^{\mathrm{f}}$ & $14.63^{\mathrm{f}}$ & $1.8 \times 10^{20}$ & 5341 & 1980 Dec 11 & 14.1 & 1795 & $2290 \pm 49$ \\
\hline
\end{tabular}

${ }^{\mathrm{a}}\left[N_{\mathrm{H}}(\right.$ Allbell $\left.)-1 \times 10^{20}\right]$ atoms cm $\mathrm{cm}^{-2}$, see text.

${ }^{\mathrm{b}}$ Gain is given on the 1-32 channel scale and represents the mean channel of the aluminum calibration line at $1.49 \mathrm{keV}$.

' In a $3^{\prime}$ radius circle.

d Bahcall, Bahcall, and Schmidt 1973.

' Battistini, Braccesi, and Formiggini 1974.

${ }^{\mathrm{f}}$ Schmidt and Green 1983.

absorption $\left(\lesssim 5 \times 10^{20}\right.$ atoms $\left.\mathrm{cm}^{-2}\right)$, and a large net total of counts $(\gtrsim 1000)$ for our first investigation of quasar spectra.

Details of the observations of three of these quasars are given in Table 1. Galactic column densities were interpolated from the Bell Labs "Allbell" Catalog (Stark et al. 1984). The IPC detections of NAB $0205+024$, B2 $1028+313$, and PG $1211+143$ have been published by Worrall and Marshall (1984), Zamorani et al. (1981), and Tananbaum et al. (1985) respectively.

For each object a fit was generated to the pulse height (PHA) channels which best covered the energy range $0.1-4.0 \mathrm{keV}$ for ranges of the parameters of various models: power law, thermal bremsstrahlung, a thermal plasma (Raymond and Smith 1977; J. Raymond 1980, personal communication), and blackbody. The absorbing column density of cold matter at cosmic abundance $N_{\mathrm{H}}$ (Brown and Gould 1970) was allowed to vary simultaneously. This fitting procedure is necessary since the broad energy resolution of the IPC precludes any unique inversion from observed counts to incident photon distribution. Contours of $\chi^{2}$ in the power-law energy index $\left(\alpha_{E}\right)$ versus $\log N_{\mathrm{H}}$ space are shown in Figure 1 for the three quasars. Formal $90 \%$ confidence intervals are given in Table 2 for a power-law fit. From Figure 1 it is clear that the allowed regions for each of the three quasars are distinct and nonoverlapping, indicating markedly different spectral characteristics for the three sources. Note that the effective gain of the IPC (Table 1) was similar in the three observations, so that any residual systematic calibration errors are not a problem. The differences between the spectra can also be seen clearly in the raw data (Fig. 2b). Residual uncertainties in the IPC calibration affect power-law fits at the level of $\sim 0.2-0.3$ in $\alpha_{E}$, as determined by cross-calibration with other instruments (particularly the SSS, the Einstein MPC, and the $H E A O$ A-2 detectors) for mutually observed AGN (Harnden and Fabricant 1985).

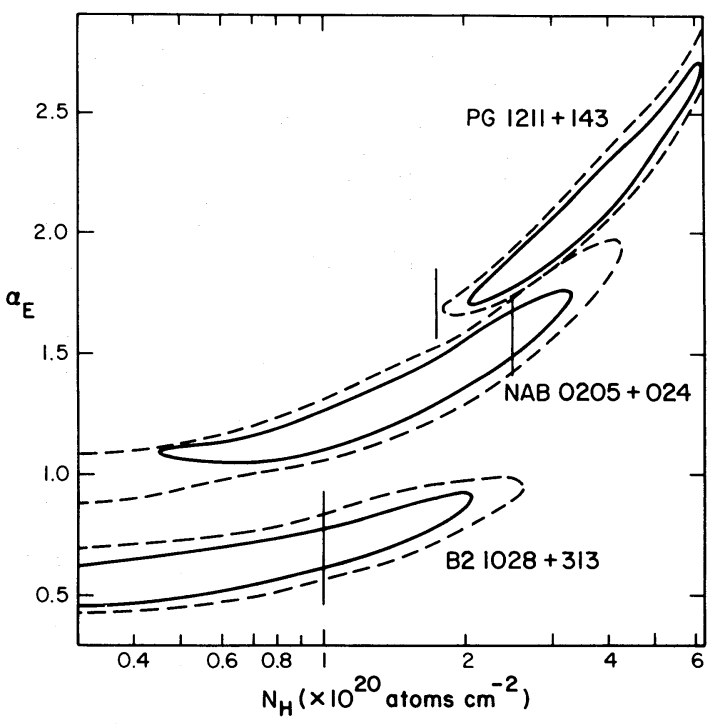

FIG. 1.-The $90 \%$ and $99 \%$ confidence regions for power-law fits to the three quasar IPC spectra are shown as the solid and dashed curves respectively. $\alpha_{E}$ is the energy spectral index $\left(f_{v} \propto v^{-\alpha}\right)$, and $N_{\mathrm{H}}$ is the allowed equivalent hydrogen column in units of $10^{20}$ atoms $\mathrm{cm}^{-2}$. The vertical lines for each quasar mark an estimate of their minimum $\mathrm{H}$ I column respectively (see § II).

Power-law fits gave acceptable minimum $\chi^{2}$ for NAB $0205+024$ and B2 $1028+313$, the two objects with flatter spectra. For PG $1211+143$, with its very steep, soft spectrum, a single power law did not give an acceptable fit. PG $1211+143$ was simultaneously detected by the MPC (Halpern 1982). Its large $5.6 \mathrm{keV}$ flux density (Fig. $2 a$ ) indicates that another, flatspectrum component is present in this quasar's X-ray emission.

TABLE 2

POWER-LaW Fits to QUaSAR X-Ray SPEctra

\begin{tabular}{cccccc}
\hline Quasar & $\alpha_{E}{ }^{\mathrm{a}}$ & $N_{\mathbf{H}}{ }^{\mathrm{a}}$ & $\begin{array}{c}\chi^{2} \text { minimum/degrees } \\
\text { of freedom }\end{array}$ & $\alpha_{E}\left[N_{\mathbf{H}}>N_{\mathbf{H}}(\text { galactic) }]^{\mathrm{a}, \mathrm{b}}\right.$ & Intrinsic $N_{\mathbf{H}}$ \\
\hline NAB $0205+024 \ldots \ldots \ldots \ldots$ & $1.2_{-0.2}^{+0.6}$ & $<3 \times 10^{20}$ & $11.4 / 7$ & $1.55_{-0.10}^{+0.25}$ & $<1 \times 10^{20}$ \\
B2 $1028+313 \ldots \ldots \ldots \ldots \ldots$ & $0.6_{-0.2}^{+0.3}$ & $<2 \times 10^{20}$ & $4.8 / 6$ & $0.7_{-0.1}^{+0.2}$ & $<1 \times 10^{20}$ \\
PG $1211+143 \ldots \ldots \ldots \ldots \ldots$ & $2.2 \pm 0.4$ & $<6 \times 10^{20}$ & $21.3 / 7$ & $2.2 \pm 0.4$ & $<4 \times 10^{20}$ \\
\hline
\end{tabular}

a $90 \%$ confidence, two parameter errors, $\left(\chi_{\text {min }}^{2}+4.6\right.$, Avni 1976).

${ }^{\mathrm{b}}\left[N_{\mathbf{H}}(\right.$ Allbell $\left.)-1 \times 10^{20}\right]$ atoms $\mathrm{cm}^{-2}$, see text. 

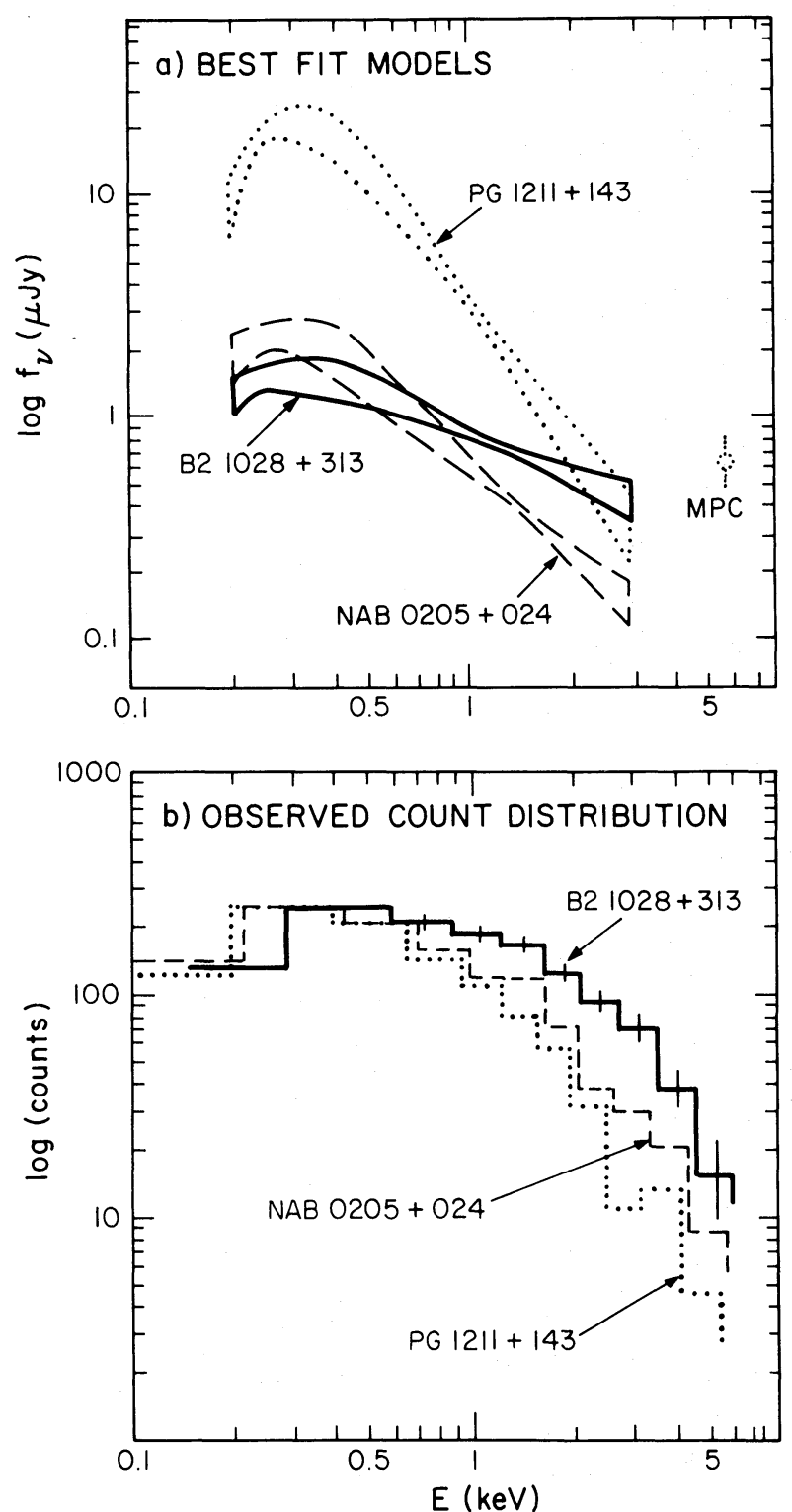

FIG. 2.- (a) The best-fit power-law models are shown for each quasar for the observed energy range. The areas show the $90 \%$ limits to the allowed $\left(\alpha_{E}\right.$, $N_{H}$ ) ranges. The point labeled MPC is the $5.6 \mathrm{keV}$ flux point for PG $1211+143$ from Halpern 1982. (b) The observed count distribution is shown for each quasar. The counts for NAB $0205+024$ and PG $1211+143$ have been normalized by 0.83 and 0.44 respectively to match B2 $1028+313$ at $0.4 \mathrm{keV}$. (The best-fit values, $[a]$, have not been renormalized.) The $1 \sigma$ error bars for B2 $1028+313$ are shown. Error bars for the other two quasars are similar.
None of the three quasars shows any evidence for a column density of cold matter in excess of the galactic value (see Tables 1 and 2). In all three cases the $90 \%$ confidence region included the galactic column density (Figure 1). However, in converting from the accurate measurements of $\mathrm{H}$ I column density to $\mathrm{X}$-ray column, assumptions as to gas-to-dust ratio and relative abundances along the line of sight are made. These add substantial uncertainties to the real X-ray column $\left(\sim \pm 1 \times 10^{20}\right.$ atoms $\mathrm{cm}^{-2}$, see, e.g., Jenkins and Savage 1974), significantly reducing their usefulness in IPC spectral fitting. We have plotted the nominal "Allbell" value minus $1 \times 10^{20}$ atoms $\mathrm{cm}^{-2}$ in Figure 1, as this imposes the least constraint on the X-ray spectra. We note that the X-ray columns for B2 $1028+313$ and NAB $0205+024$ are only just consistent with the nominal $N_{\mathbf{H}}$ (galactic) values.

The other three simple spectral forms (thermal bremsstrahlung, thermal plasma, and blackbody) all gave larger $\chi^{2}$ than the power law and required column densities below $1 \times 10^{20}$, inconsistent with the minimum required galactic columns. Of these three a thermal bremsstrahlung fit came closest to giving an acceptable fit for all three quasars. A formal $F$-test comparing the minimum $\chi^{2}$, with the condition that $N_{\mathrm{H}} \geq 1 \times 10^{20}$ atoms $\mathrm{cm}^{-2}$, can reject a blackbody fit for NAB $0205+024$ and B2 $1028+313$ and also rejects a Raymond thermal plasma for PG $1211+143$. For simplicity we shall use the power-law fits in the following discussion. In Table 3 the corresponding broad-band and monochromatic fluxes are given for the standard IPC $0.3-3.5 \mathrm{keV}$ band and for two energies of particular interest, $0.2 \mathrm{keV}$ and $2 \mathrm{keV}$.

\section{DISCUSSION}

The derived IPC spectra demonstrate that there is no single universal power-law slope for quasar X-ray spectra. Indeed, a wide diversity exists within the X-ray spectra of quasars. Power-law models give best fits which range from 0.6 to 2.2 in energy index. In addition, a single power-law model does not always provide a good fit to the data, so that the above statement oversimplifies the real situation. Even when a single power law plus column provides an acceptable fit, the possibility that two components are contributing cannot be ruled out. Such an interpretation is supported for PG $1211+143$ by MPC observations which indicate a flatter slope at higher energies than the fit to the IPC data predicts.

Our limits on the intrinsic column densities toward quasars are a factor of 5-10 times lower than the best previous limits (Petre et al. 1984) for cold material at cosmic abundances. They correspond to an optical reddening $A_{v} \lesssim 0.1$ (Jenkins and Savage 1974). We can thus exclude that we are viewing these quasars through the disk of a host or intervening normal spiral

TABLE 3

Derived Quasar Fluxes and Luminosities for Power-Law Fits

\begin{tabular}{ccccc}
\hline \hline Quasar & $\begin{array}{c}f(0.2 \mathrm{keV}) \\
(\mu \mathrm{Jy})\end{array}$ & $\begin{array}{c}f(2 \mathrm{keV}) \\
(\mu \mathrm{Jy})\end{array}$ & $\begin{array}{c}f(0.3-3.5 \mathrm{keV}) \\
\left(\mathrm{ergs} \mathrm{cm}^{-2} \mathrm{~s}^{-1}\right)\end{array}$ & $\begin{array}{c}L_{x}(0.3-3.5 \mathrm{keV}) \\
\left(\mathrm{ergs} \mathrm{s}^{-1}\right)\end{array}$ \\
\hline NAB $0205+024 \ldots \ldots \ldots \ldots \ldots$ & $5.4_{-2.2}^{+6.0}$ & $0.220_{-0.01}^{+0.02}$ & $\left(3.5_{-0.3}^{+1.2}\right) \times 10^{-12}$ & $\left(4.9_{-0.4}^{+1.7}\right) \times 10^{44}$ \\
B2 $1028+313 \ldots \ldots \ldots \ldots \ldots$ & $2.1_{-0.46}^{+1.7}$ & $0.50_{-0.03}^{+0.06}$ & $\left(5.0_{-0.3}^{+0.3}\right) \times 10^{-12}$ & $\left(8.1_{-0.48}^{+0.48}\right) \times 10^{44}$ \\
PG $1211+143 \ldots \ldots \ldots \ldots \ldots$ & $200_{-130}^{+70}$ & $0.78_{-0.07}^{+0.25}$ & $\left(3.7_{-0.2}^{+0.5}\right) \times 10^{-11}$ & $\left(1.3_{-0.07}^{+0.17}\right) \times 10^{45}$ \\
\hline
\end{tabular}

NoTE.-All fluxes are corrected for galactic absorption by the fitted $N_{\mathrm{H}}$ values of Table 2 . They are given in the energy frame of the observer. 
galaxy. Any further advance in measuring small X-ray column densities must come from improved spectral resolution, since the uncertainty on our own galactic column density is now the dominant uncertainty. This is also true for interesting cases of hot $\left(T>10^{5} \mathrm{~K}\right)$ intervening materials, since most of our information on column density comes from the energy range below the $0.28 \mathrm{keV}$ carbon edge. It is thus dependent largely on the helium opacity (column densities large enough to be affected substantially by oxygen opacity restrict the effective IPC bandpass enough to preclude useful spectral fits). Since helium is largely ionized above a few $10^{5} \mathrm{~K}$, our columns do not restrict conditions in hot intervening matter such as an intergalactic medium (Shapiro and Bahcall 1980) or an intercloud medium for the emission-line regions (Krolik, McKee, and Tarter 1981). However, the lack of large column densities of cold matter means that these quasars will be good candidates for future high-resolution searches for these hot media.

The observed diversity in power law spectral index is important to the study of quasars in many ways, three of which are discussed below.

\section{a) X-Ray Background}

In assessing the contribution of quasars to the X-ray background over the IPC energy range, a monochromatic flux at 2 $\mathrm{keV} f(2 \mathrm{keV})$ has normally been derived from the total count rate in the energy band by assuming a universal index of 0.5 (Tananbaum et al. 1979; Zamorani et al. 1981; Marshall et al. $1983,1984)$. Since $2 \mathrm{keV}$ is close to the high end of the range, a steeper index will lower the derived $f(2 \mathrm{keV})$ for a given count rate. If the mean index of quasar spectra is steeper than 0.5 , as our results suggest, then the quasar contribution to the X-ray background at $2 \mathrm{keV}$ will be lowered. At $2 \mathrm{keV}$ for $N_{\mathrm{H}} \approx 3$ $\times 10^{20}$ atoms $\mathrm{cm}^{-2}$, the flux is reduced by $\sim 40 \%$ when the index is changed from 0.5 to 1.2 . Thus, for example, the $2 \mathrm{keV}$ background contribution given by Marshall et al. (1984) for UV excess quasars with $M_{b}<19.8$ and $z<2.2$ was $24_{-8}^{+10} \%$. This would become $14_{-5}^{+6} \%$. These are significant but not overwhelming differences. The broad-band fluxes $(0.3-3.5 \mathrm{keV})$ would also be affected, but by a lesser amount, with a reduction of about $15 \%$. However, the impact on calculations using optical luminosity and evolution functions is not so directly assessed, since these calculations make use of the X-ray-tooptical luminosity ratio and the effect on X-ray luminosity of changing X-ray spectral index depends on redshift due to $K$ correction effects. Clearly some knowledge of the X-ray spectral slope in individual cases is essential in determining the contribution of quasars to the X-ray background even at a single energy.

\section{b) X-Ray Continuum Emission Mechanisms}

Comparisons with models, most notably the synchrotron self-Compton (SSC) model, generally use the slope $\alpha_{o x}$ from the optical to the X-ray as a measure of the relative strength of optical and X-ray emission. The X-ray flux is normally assumed to be inverse Compton emission in this model. Our data now make it clear that a measured X-ray spectrum is essential in determining the continuum emission mechanism in each individual case. The three spectra presented here may be interpreted in terms of the different mechanisms possible in the SSC model: B2 $1028+313$ with its flat $\sim 0.6$ slope can be identified with the inverse Compton component; NAB $0205+024$, with a slope of $\sim 1.2$ could be a direct extrapolation of the optical-infrared synchrotron continuum; and PG
$1211+143$ with its steep slope of $\sim 2.2$ may be showing us the high energy cutoff of the synchrotron spectrum. However, since the SSC model can give any slope steeper than 0.7 , no observed slope tests this model. At this stage other emission processes (e.g., thermal comptonization, Maraschi, Roasio, and Treves 1982; coronal emission from an accretion disk, Galeev, Rosner, and Vaiana 1979) are equally plausible to explain the observed "soft excess" in quasars.

These results support the suggestion of Zamorani et al. (1981) that more than one X-ray emission mechanism must be operating in quasars. This suggestion was based on the observation that radio-loud quasars have systematically more X-ray emission for a given optical magnitude, by a factor of 3 (i.e., smaller $\alpha_{o x}$ ), than radio-quiet quasars. A larger sample of radioloud quásars is needed to determine whether their X-ray spectra are normally flatter than those of radio-quiet objects.

\section{c) Emission Lines}

The X-ray continuum plays two major roles in the broad emission-line region of quasars: first in the formation and confinement of the emitting clouds; and second in their ionization.

Hard X-ray heating of the atmosphere around a quasar can result in a two-phase instability producing the broad emissionline clouds and a hot confining medium $\left(T_{h} \approx 10^{8} \mathrm{~K}\right.$, Krolik, McKee, and Tarter 1981). Our results indicate that this model may be too simple. A strong, steep X-ray continuum with $\langle h v\rangle\left\langle k T_{h}\right.$, such as that of PG $1211+143$, leads to Compton cooling of this atmosphere, which is likely to remove the instability and thus the emitting clouds (Guilbert, Fabian, and McCray 1983). However, in order to be in the Schmidt and Green (1983) catalog, PG $1211+143$ must possess strong, broad emission lines. Clearly, an additional heating source is needed to maintain the instability, such as a weaker, hard $\mathrm{X}$-ray component extending to high energies. This may have been seen in the MPC measurements. Alternatively, adiabatic heating may provide the necessary energy input if the quasar atmosphere is accreting ( $\mathrm{J}$. Krolik, private communication). Detailed calculations for specific objects such as PG $1211+143$ are needed.

The soft X-ray spectrum also provides the best available constraints on the continuum responsible for the bulk of the ionization in the emission-line clouds. The flux at $2 \mathrm{keV}$ (Table 3) and above is believed to be responsible for the production of low ionization lines (e.g., $\mathrm{Mg}$ II, Fe II) deep within the broadline clouds (Kwan and Krolik 1981). The flux around $0.2 \mathrm{keV}$ (Table 3 ) is largely responsible for the very high ionization lines (e.g., Ne v, Fe vII, X, XI, XIV, which have ionization potentials at $0.10,0.10,0.24,0.26$, and $0.36 \mathrm{keV}$ respectively), although collisional ionization may also contribute (Osterbrock 1981; Penston et al. 1984). Direct measurements of soft X-ray continuum fluxes will shed more light on this issue.

\section{CONCLUSIONS}

This preliminary study raises issues stemming from the observation of diverse soft X-ray spectra in quasars. At this stage we do not know the relative frequency with which the different spectra are found, nor do we know whether they form a continuous distribution of slopes or fall into several welldefined groups.

The small values for the allowed intrinsic column density of cold matter in these quasars are almost an order of magnitude smaller than previous limits. These data do not constrain hot $\left(T>10^{5} \mathrm{~K}\right)$ media significantly. However, the lack of any con- 
fusing cold absorbing material makes these quasars good candidates for future searches for hot media at high spectral resolution.

As the IPC reprocessing continues, we shall expand our study. We estimate that there may be 50 quasar spectra of reasonable quality to be derived from the IPC data base. These will include the X-ray-bright members of two well-defined samples: UV-excess selected PG quasars (Schmidt and Green 1983) and low-frequency selected radio-loud 3CR quasars (Tananbaum et al. 1983). Unfortunately, particularly for those concerned with the X-ray background, no sample of X-rayselected quasars was observed on axis with the IPC. Since quasar absorbing columns seem to be small, this gap may be filled by future low-energy observations during the ROSAT mission. The higher resolution detectors, high-energy response, and larger collecting area of AXAF will allow study of the deviations from our näive power-law assumption for quasar X-ray spectra.

We are grateful to $\mathbf{M}$. Schmidt and $\mathbf{R}$. Green for permission to use the data on PG $1211+143$. The data for NAB $0205+024$ came from the Einstein data bank. We thank F. R. Harnden and his team for their heroic calibration effort on the IPC. We also thank Julian Krolik for useful discussions, and Claude Poux and John Janetos for valuable assistance with the data analysis.

This work was supported by NASA contract NAS8-30751.
Avni, Y. 1976, Ap. J., 210, 642

Bahcall, N. A., Bahcall, J. N., and Schmidt, M. 1973, Ap. J., 183, 777.

Battistini, P., Braccesi, A., and Formiggini, L. 1974, Astr. Ap., 35, 93.

Brown, R. L., and Gould, R. 1970, Phys. Rev., D2, 2252.

Galeev, A. A., Rosner, R., and Vaiana, G. S. 1979, Ap. J., 229, 318.

Giacconi, R., et al. 1979, Ap. J., 230, 540.

Gioia, I. M., Maccacaro, T., Schild, R. E., Stocke, J. T., Liebert, J. W., Danziger, I. J., Kunth, D., and Lub, J. 1984, Ap. J., 283, 495.

Gorenstein, P., Harnden, F. R., and Fabricant, D. G. 1981, IEEE Trans., NS-28, 869

Guilbert, P. W., Fabian, A. C., and McCray, R. 1983, Ap. J., 266, 466

Halpern, J. P. 1982, Ph.D. thesis, Harvard University.

Harnden, F. R., Jr., and Fabricant, D. 1985, in preparation.

Harnden, F. R., Jr., Fabricant, D. G., Harris, D. E., and Schwarz, J. 1984, Smithsonian Ap. Obs. Spec. Rept., No. 393.

Jenkins, E. B., and Savage, B. D. 1974, Ap. J., 187, 243.

Kazanas, D. 1984, Photon-Photon Absorption and the Uniqueness of the Spectra of Active Galactic Nuclei (NASA TM-86117).

Kriss, G. A., and Canizares, C. R. 1982, Ap. J., 261, 51.

Krolik, J. H., McKee, C. F., and Tarter, C. B. 1981, Ap. J., 249, 422.

Ku, W. H.-M., Helfand, D. J., and Lucy, L. B. 1980, Nature, 288, 323.

Kwan, J. Y., and Krolik, J. H. 1981, Ap. J., 250, 478.

Lawrence, A., and Elvis, M. 1982, Ap. J., 253, 410.

Lightman, A. P. 1981 , in X-Ray Astronomy in the 1980 's, ed. S. S. Holt, NASA Technical Memorandum 83848, p. 143

Maccacaro, T., Gioia, I. M., and Stocke, J. 1984, Ap. J., 283, 486.

Maraschi, L., Roasio, R., and Treves, A. 1982, A p. J., 253, 312

Margon, B., Chanan, G. A., and Downes, R. A. 1982, Ap. J. (Letters), 253, L7.

Marshall, H. L., Avni, Y., Braccesi, A., Huchra, J. P., Tananbaum, H., Zamorani, G., and Zitelli, V. 1984, Ap. J., 283, 50.

\section{REFERENCES}

Marshall, H. L., Avni, Y., Tananbaum, H., and Zamorani, G. 1983, Ap. J., 269, 35.

Mezaros, P. 1983, Ap. J. (Letters), 274, L13.

Mushotzky, R. F. 1982, Ap. J., 256, 92.

- 1984, Adv. Space Res., 3, Nos. 10-12, 157.

Osterbrock, D. E. 1981, Ap. J., 246, 696.

Penston, M. V., Fosbury, R. A. E., Boksenberg, A., Ward, M. J., and Wilson, A. S. 1984, M.N.R.A.S., 208, 347.

Petre, R., Mushotzky, R. F., Krolik, J. H., and Holt, S. S. 1984, Ap. J., 280, 499.

Pravdo, S. H., Nugent, J. J., Nousek, J. A., Jensen, K., Wilson, A. S., and Becker, R. H. 1981, Ap. J., 251, 501.

Raymond, J., and Smith, B. W. 1977, Ap. J. Suppl., 35, 419.

Rees, M. J. 1981, Space Sci. Rev., 30, 87.

Reichert, G. A., Mason, K. O., Thorstensen, J. R., and Bowyer, S. 1982, Ap. J., 260, 437.

Rothschild, R. E., Mushotzky, R. F., Baity, W. A., Gruber, D. E., Matteson, J. L., and Primini, F. A. 1983, Ap. J., 269, 423.

Schlossman, I., Shaham, J., and Shaviv, G. 1984, Ap. J., 287, 534

Schmidt, M., and Green, R. F. 1983, Ap. J., 269, 352.

Shapiro, P. R., and Bahcall, J. N. 1980, Ap. J., 241,

Stark, A. A., Heiles, C., Bally, J., and Linke, R. 1984, in preparation.

Stocke, J. T., Liebert, J., Gioia, I. M., Griffiths, R. E., Maccacaro, T., Danziger, I. J., Kunth, D., and Lub, J. 1983, Ap. J., 273, 458.

Tananbaum, H., et al. 1979, Ap. J. (Letters), 234, L9.

Tananbaum, H., Avni, Y., Green, R. F., Schmidt, M., and Zamorani, G. 1985, in preparation.

Tananbaum, H., Wardle, J. F. C., Zamorani, G., and Avni, Y. 1983, Ap. J., 268, 60.

Worrall, D., and Marshall, F. E. 1984, Ap. J., 276, 434

Zamorani, G., et al. 1981, Ap. J., 245, 357.

Martin Elvis, Harvey Tananbaum, and Belinda J. Wilkes: Harvard-Smithsonian Center for Astrophysics, 60 Garden Street, Cambridge, MA 02138 